\title{
La integración en las pequeñas y medianas empresas fabricantes de muebles de la ciudad de Durango, México
}

\section{Francisco Martín Villarreal Solís* José Gerardo Ignacio Gómez Romero**}

* Profesor de la Universidad Juárez del Estado de Durango. Correo electrónico: fmvillasol@yahoo.com.mx

** Profesor de la Universidad Juárez del Estado de Durango Correo electrónico: gerardoignaciog@yahoo.com.mx

\section{Resumen}

Las pequeñas y medianas empresas en todo el mundo comparten dos características: constituyen porcentualmente el segundo segmento en cuanto a número de empresas; y manifiestan diversas debilidades derivadas de su tamaño.

Una estrategia que se puede emplear para transformar las debilidades en fortalezas es la integración, que a su vez puede darse en dos dimensiones: vertical y horizontal, las cuales permiten a las empresas controlar las diferentes etapas de su cadena de valor y realizar alianzas estratégicas con otras empresas competidoras para, así, lograr entre todos una sinergia que beneficie a los participantes de esta unión.

Nuestro estudio exploró la industria mueblera en la ciudad de Durango, México, seleccionada por su importancia socioeconómica en la localidad y determinada por su vocación forestal histórica. Por otra parte, se realizó un diagnóstico de la situación de la integración mediante el diseño de un instrumento que consideró el tipo de integración que emplean las empresas y en qué grado de acuerdo con varias dimensiones; así como la posible influencia de ésta en la permanencia y la rentabilidad, la caracterización de las empresas muebleras y de los empresarios que las poseen. 
Palabras clave: integración vertical, integración horizontal, rentabilidad, permanencia, alianza estratégica, cadena de valor.

\title{
Integration in small and medium size enterprises in the furniture manu- facturing sector of Durango, Mexico
}

\begin{abstract}
Small and medium enterprises around the world share two characteristics: they are the most numerous and they have many size-related problems. Integration is a strategy that can be used to transform these weaknesses into strengths and it can be analyzed in two dimensions: vertical and horizontal. Integration can be used to control the different stages of the value chain and to make strategic alliances with the competition to achieve a synergy that benefits every participant. The present study explores the furniture industry of Durango because of the importance of the forest-related industries in the local context. An instrument was developed in order to diagnose integration which took into consideration the type and extent of integration shown by the companies, its possible influence on their permanence and profitability, and the characterization of the enterprises and their owners.
\end{abstract}

Key words: Vertical integration, horizontal integration, profitability, permanence, value chain, strategic alliances.

\section{Introducción}

\subsection{Introducción al problema}

El propósito de este trabajo es explorar y analizar el efecto que tienen la integración vertical y horizontal en la permanencia y rentabilidad de las Pymes fabricantes de muebles de la capital del estado de Durango. En este sentido, se realizó un diagnóstico exploratorio de la integración en estas empresas en cuanto al tipo y grado de la misma.

Para ello, se acotó el universo a las pequeñas y medianas empresas debido a la importancia que tienen; por ejemplo, la Secretaría de Economía (2004) difunde en la página electrónica del Sistema Empresarial (Siem) que en México las pequeñas y medianas empresas (Pymes) representan el $13.77 \%$ del total de las empresas 
establecidas y que generan el $4.8 \%$ de los empleos en México; lo anterior, frente al $0.2 \%$ que crea la gran empresa (las que emplean a más de 250 trabajadores). De acuerdo con la misma fuente, la mayoría de las empresas y el empleo en el país lo aportan las microempresas.

Un argumento que muestra la importancia de estas empresas es el presentado por Dallago (2003) en el sentido de que el sector de las empresas medianas y pequeñas apuntala la transformación de la economía al generar empleos y al ayudar a la adaptación e innovación del sistema económico lo que indica lo necesarias que son para el crecimiento económico de un país, de ahí que necesiten todo el apoyo que se les pueda otorgar, tanto por parte del gobierno como de los académicos, estudiando los factores que les permitan ser rentables y permanecer.

Esta situación ha generado varios trabajos acerca de las Pymes por diversos autores: Audretsch (1991), Audretsch y Zoltan (1990), Audretsch y Mahmood (1994) (1995), Camisón (1997) y (2001), Lafuente y Yagüe (1989), Sexton y Van Auken (1985), Tirado (1995), Watson y Everett (1996) por mencionar algunos solamente; a este interés del mundo académico se le agrega la gran atención por parte del gobierno en todos sus niveles, cuyos titulares han tomado diversas medidas para su fomento y desarrollo.

Este trabajo se adhiere a esta corriente para analizar una situación que se presenta en muchas empresas de diversos sectores: el uso de la integración como estrategia; asimismo, es concebido por la idea de que contribuirá a aumentar el conocimiento acerca de las pequeñas y medianas empresas, específicamente, amén de convertirse en generador de alternativas de acción para los administradores y propietarios de las mismas.

\subsection{Planteamiento del problema}

En resumen, nuestro trabajo pretende determinar si hay diferencias entre las empresas en rentabilidad y permanencia, de acuerdo con el tipo y grado de integración que empleen.

La adopción de la integración como estrategia dependerá en términos generales de las condiciones del medio considerando que en ocasiones, según la evidencia empírica, ha resultado ser benéfica para las empresas; mientras que, en función de los resultados a largo plazo, en otras no ha sido así. Sin embargo, de acuerdo con 
Hernández Romo (2004), es importante considerar que no sólo la influencia del entorno es relevante para la adopción de alguna estrategia (como la integración), sino que desde luego cuenta la propia voluntad del empresario.

\subsection{Objetivos y preguntas de investigación}

\section{Objetivos:}

Determinar los tipos de integración que utilizan las pequeñas y medianas empresas del sector mueblero de la capital del estado de Durango como estrategia para enfrentar los retos del mercado.

Analizar el comportamiento de la rentabilidad de las pequeñas y medianas empresas del sector mueblero de la capital del estado de Durango, de acuerdo con el tipo de integración que empleen.

Analizar la relación que existe entre el tipo de integración que usen las pequeñas y medianas empresas del sector mueblero de la capital del estado de Durango y su permanencia en el tiempo.

Preguntas de investigación:

¿Qué tipos de integración utilizan las pequeñas y medianas empresas del sector mueblero de la capital del estado de Durango? ¿Cuál es el comportamiento de la rentabilidad de las pequeñas y medianas empresas del sector mueblero de la capital del estado de Durango de acuerdo con el tipo de integración que empleen? ¿Cuáles son los efectos en la permanencia en el tiempo de las pequeñas y medianas empresas del sector mueblero de la capital del estado de Durango según el tipo de integración que se usa?

\subsection{Hipótesis}

Para el estudio se plantearon las siguientes hipótesis de investigación. Hipótesis: Las pequeñas y medianas empresas del sector mueblero de la capital del estado de Durango utilizan la integración horizontal y/o vertical, lo cual tiene efectos positivos en su permanencia y rentabilidad. 
Hipótesis particular 1: Las pequeñas y medianas empresas del sector mueblero de la capital del estado de Durango emplean la integración horizontal y/o vertical.

Hipótesis particular 2: A mayor integración vertical y/o horizontal mejor rentabilidad en las empresas estudiadas.

Hipótesis particular 3: A mayor integración vertical y/o horizontal mayor permanencia en las empresas estudiadas

\subsection{Justificación}

Para complementar la importancia económica de la pequeña y mediana empresa, es oportuno citar a Rojas (1995) quien señala que uno de los principales retos del sector forestal en México y Centroamérica es el de desarrollar modelos de auténtica integración del bosque con la industria porque no es prudente, estratégico ni recomendable concebirlos como entes productivos aislados. Este reto llama entonces al fomento de la integración vertical y horizontal en el sector; sin embargo, sobre ello aún hay que investigar, lo que justifica en parte este proyecto.

\section{Literatura revisada}

En términos generales, la estrategia es la forma en la que se habrán de alcanzar los objetivos de las organizaciones en una situación de competencia; asimismo, se puede definir como el patrón o modelo de decisiones que determina y revela sus objetivos, propósitos o metas. Por otra parte, produce las políticas y planes necesarios para alcanzar dichas metas (Andrews, 1965, como citado por Mintzberg y Quinn, 1993).

Considerando la problemática que experimentan las Pymes en la actualidad, misma que puede obstaculizar el logro de sus metas, es necesario entonces recurrir a estrategias competitivas que permitan alcanzar una ventaja respecto a sus competidores, considerando que en estos tiempos ellos pueden localizarse al otro lado del mundo debido al proceso de globalización que ha caracterizado a los mercados contemporáneos. 
Una de estas estrategias es la integración, que está destinada a incrementar las utilidades de las empresas mediante la mejora de los procesos de creación de valor y el aumento en la rentabilidad, lo cual conduce a mejores oportunidades de permanencia. La integración, por otra parte, se da en dos modalidades: la vertical, donde la empresa trata de controlar las etapas sucesivas de la cadena de valor del producto, y la horizontal, en la cual en una misma etapa de la cadena de valor se fomentan la cooperación y las alianzas estratégicas con los competidores. Ambas integraciones son estrategias válidas y pueden usarse por separado o conjuntamente. Sin embargo, Colangelo (1995:2) en su estudio señala que "la ganancia total de la integración vertical es frecuentemente mayor que la de la integración horizontal”.

De acuerdo con Cai y Obara (2005) la integración horizontal es muy común en industrias que proveen servicios y en donde la interacción cliente-empresa es poco frecuente, por ejemplo: hoteles, renta de automóviles y tiendas de conveniencia; en estos casos, tiene un impacto positivo en la reputación de las firmas. Este tipo de integración, de acuerdo con Hill y Jones (2005) puede aumentar la rentabilidad de las empresas a través de reducción de costos, lo cual puede ser por el incremento en las economías de escala, aumento de valor, por la posibilidad de ofrecer más productos mediante el agrupamiento; es decir, ofrecer un conjunto de productos pero cobrar un solo precio, manejar de la competencia en la industria mediante la eliminación de la capacidad excesiva y las guerras de precios, aumentar de la capacidad de negociación con proveedores y clientes.

Una de las limitaciones de la integración horizontal es que no siempre se crea valor, sino al contrario: puede tener efectos adversos tanto para las empresas como para los clientes. Algunas consecuencias de la integración horizontal van del monopolio al oligopolio que se darán si las empresas que controlan una industria se alían creando un mercado con poca o nula competencia, o si las culturas organizacionales son muy divergentes; por ejemplo, si una organización es muy conservadora en sus políticas de personal y la otra tiene políticas liberales; si la adquisición fue hostil, situación que a veces se da cuando la empresa dominante fuerza la situación para adquirir influencia sobre la más débil; o si no hubo transparencia en las operaciones financieras donde el proceso puede desvirtuarse y quedar anulado o, incluso si perjudicara la rentabilidad como consecuencia de la escasa compatibilidad entre ambas para colaborar juntas y para fijar objetivos de realización común. 
Acerca de la integración horizontal en nuestro país se han llevado a cabo pocos estudios. Gómez (2000) en un estudio acerca de la industria química, investigó la capacidad de ésta para establecer alianzas estratégicas y propuso una tipología de las empresas, aunque al final sus conclusiones fueron poco halaguieñas porque encontró que de 13 empresas que declararon tener alianzas estratégicas, sólo 5 registraron finalmente llevarlas a cabo. Es también significativo que las empresas únicamente deseen recibir maquinaria y equipo y no estén interesadas en el desarrollo de tecnología ni de patentes.

Según Mpoyi (2003) la integración vertical puede verse como el grado en que una firma controla la producción de sus insumos o abastecimientos y la distribución de sus productos terminados. En el primer caso se presenta una integración vertical para atrás y en el segundo se trata de una integración vertical para adelante.

Otra definición de integración vertical es la que señala Paredes (1997) quien menciona que las organizaciones de tipo vertical son aquellas que se encuentran relacionadas patrimonialmente en sus etapas insumo-producto. El autor enfatiza que a diferencia de otros países cuyas experiencias muestran que hay mucho que aprender y conocer en esta materia (las organizaciones integradas verticalmente); afirma que en Chile a nivel conceptual es común dar por terminado el debate y generalmente se concluye de manera errónea que la integración vertical tiene un costo social; situación por la que invita a proseguir el debate acerca de la integración y su regulación. Por su parte, Galetovic (2003) menciona que la integración vertical en el sector eléctrico es en los últimos años un tema muy discutido después de dos décadas de privatizaciones y liberalizaciones alrededor del mundo.

Finalmente, Gil (2005) en su estudio sobre la integración vertical en la industria cinematográfica, reconoce que la evidencia empírica en los efectos de la integración vertical permanece escasa debido a que en mucha de la literatura empírica se examina cómo las formas organizacionales cambian en función de las características de las transacciones que efectúan y, por ello, exploran la integración sólo indirectamente. En ese sentido, la cuestión de cómo y en qué grado la integración afecta los resultados económicos permanece inexplorada.

Un concepto básico en la integración vertical es la cadena de valor (Porter, 1990) que es precisamente la secuencia de actividades y procesos en los que se va adicionando valor al producto; en la integración vertical, lo que se pretende es acceder a esas etapas de creación de valor. 
Por ejemplo, la empresa forestal que decide, en lugar de comprar la madera ya estufada ${ }^{1}$, invertir en una estufa estará haciendo integración vertical hacia atrás en su cadena de valor. Si en cambio decide hacerse de una flotilla de vehículos para entregas, la integración vertical será hacia delante.

Economides (1998) presenta algunas razones que tienen las empresas para integrarse:

a) Mejor coordinación entre componentes; hay beneficios en el uso conjunto.

b) Ahorros de costo en la producción conjunta.

c) Posibilidad de tener un mejor diseño integrado.

d) Flujos de información más rápidos en una compañía integrada.

e) Mercados más seguros para los componentes del producto.

f) Se facilita la expansión vertical a nuevos componentes o características del producto.

Por su tipo, tamaño y localización geográfica, muchas de estas razones son válidas para el tipo de empresa que se estudia en este trabajo. Las empresas manufactureras forestales recurren a estas razones para mejorar su proceso productivo, reducir sus costos, fortalecer su posición en el mercado y mejorar su distribución.

\subsection{Explicando la integración}

Desde una perspectiva económica, se puede explicar a la integración mediante la Teoría de la Firma de Coase (1939, citado por Paredes 1997) sobre el origen y naturaleza de la firma, en la cual se visualiza fácilmente el proceso de la integración. Esta teoría admite que en determinados escenarios, el uso del mercado como proveedor de recursos es muy costoso, por lo cual es conveniente sustituirlo por una opción más eficiente.

Asimismo, esta teoría tiene dos hipótesis básicas. La primera sostiene que la organización óptima de cualquier actividad es la que minimiza los costos de transacción, cuyo monto depende de las características de la transacción, especialmente la incertidumbre (a mayor incertidumbre, mayores costos) y la complejidad (mayor

\footnotetext{
${ }^{1}$ Estufar es un modismo de la industria forestal y se refiere al proceso de secar la madera en estufas sometiéndola a calor y vapor para lograr estabilizar su contenido de humedad en porcentajes variables, según la especie de madera y el uso que se le pretende dar. Asimismo, en algunas certificaciones forestales es garantía de que la madera está libre de esporas, parásitos y otros agentes dañinos.
} 
complejidad, mayores costos). El problema surge cuando una de las dos partes signatarias de un contrato tiene que efectuar una inversión alta en activos y la parte que no invirtió se aproveche de ello, con lo que se creará una situación inestable donde se generan los verdaderos costos de la transacción. En ocasiones, cuando lo anterior sucede, la transacción puede no realizarse y entonces se presenta una falla de mercado a la que se denomina hold up. La conclusión es que cuando los activos son muy específicos se recurre a la integración vertical porque genera una estructura óptima para minimizar los costos de transacción (Riordan, Williamson y Klein, citados por Compés, 2005).

Una definición clara de los costos de transacción es la proporcionada por Puig (2001), en la cual dice que toda transacción tiene costos más allá del precio de la misma. Éstos se pueden identificar con los costos que corresponden a la especificación y redacción de contratos, la evaluación de ofertas, la negociación del contrato con el proveedor elegido y el seguimiento y evaluación de los resultados del contrato.

Williamson (citado por Paredes Molina, 1997) afirma que los costos de transacción provienen de la coexistencia de tres factores: a) que no se puede prever el futuro, por lo que los contratos serán necesariamente incompletos e imperfectos; b) que no se puede tener absoluta confianza en los acuerdos o contratos; c) que las inversiones tengan un grado de especificidad a terceros, lo cual permite, por ejemplo, a un transportista subir el precio de sus servicios sabiendo que el productor no puede dejar perder su producción.

Desde esta perspectiva, es fácil comprender una de las mayores ventajas de la integración: la eliminación de los costos de transacción. Adicionalmente, al controlar la cadena de valor se reduce a la competencia.

En la actualidad el debate respecto a la integración se encuentra, en su mayor parte, en las áreas de economía y matemáticas aplicadas porque, de acuerdo con Scazzieri (1990), sólo recientemente el concepto de integración vertical ha sido rigurosamente identificado y sus propiedades lógicas sometidas a investigación teórica explícita; sin embargo, recordemos las bases teóricas vienen inclusive desde el siglo XVIII con Adam Smith.

Las implicaciones teóricas de la integración han representado un importante campo en el debate teórico, al menos desde que surgió una teoría de la integración vertical de los recursos productivos (Clark, 1899, como citado por Scazzeri, 1990) 
y la controversia sobre los méritos relativos de las diversas representaciones del proceso productivo.

\section{Metodologia}

Las variables que se consideraron en el estudio son las siguientes: 1. Integración horizontal, 2. Integración vertical, 3. Permanencia en el tiempo, y 4. Rentabilidad.

En este caso, al no encontrar en la literatura un instrumento que explorara los aspectos considerados necesarios, se diseñó uno que recauda la información de las tres variables mediante cinco secciones:

- Cadena de valor, donde se pidió al empresario que describiera las etapas de la cadena de valor de su negocio, mediante cuatro reactivos que midieron el conocimiento de las etapas por las que atraviesa la materia prima hasta convertirse en producto terminado.

- Integración horizontal, donde se determinó si existe y en qué grado, con cinco reactivos que se aplicaron para constatar cómo progresivamente aumenta la colaboración de la empresa con sus competidores, los cuales son: 1) ninguna relación en absoluto; 2) relación informal ocasional; 3) relación informal orientada a acceder en conjunto con los competidores a determinadas tecnologías, procesos, fuentes de materia prima, etc.; 4) acuerdos formales que están funcionando en la práctica para lograr mejores condiciones en el acceso a fuentes de materia prima, mercados, canales de distribución, tecnologías o procesos; $y$, finalmente, 5) la existencia de alianzas estratégicas formales que involucran fusiones o adquisiciones o participación en otras empresas.

- Integración vertical, que buscó determinar si existe y en qué grado, con cinco reactivos que miden cómo aumenta progresivamente el control de la empresa sobre las diversas etapas de la cadena de valor, que son: 1) la empresa realiza todas sus compras de materiales primas a proveedores y hace sus ventas a distribuidores o mayoristas, es decir, se concentra únicamente en la actividad principal de su negocio; 2) la empresa ha hecho esfuerzos para asegurarse el flujo de materia prima o la salida de sus productos adquiriendo activos para usarlos en esas actividades; 3 ) la empresa realiza 
cotidianamente actividades relativas al control y operación ya sea del acceso a las materias primas o del acceso a los mercados del producto terminado; 4) la empresa ha realizado inversiones considerables para controlar el abastecimiento de materias primas, su traslado, procesamiento, distribución y venta del producto terminado, y ha dejado de depender de terceros casi por completo; y 5) cuando la empresa controla completamente la cadena de valor del producto, desde la producción de la materia prima básica hasta la venta al consumidor final a través de sus propios canales de distribución.

- Permanencia, donde se recopiló información acerca de la supervivencia en el tiempo de la empresa, preguntando cuál es la antigüedad de la misma y mediante el establecimiento de cinco dimensiones de permanencia. Asimismo, se incluyeron preguntas relativas al número de trabajadores y el comportamiento de este número.

- Rentabilidad, donde se buscó la información acerca de la capacidad de la empresa para generar utilidades al preguntar acerca del margen de utilidad neta del ejercicio anterior y el comportamiento de este margen respecto al pasado.

Para llevar a cabo lo anterior se efectuó, de inicio, una prueba piloto con cinco empresas correspondientes a las categorías de pequeña y media (de acuerdo con el criterio publicado en el Diario Oficial de la Federación del 30 de diciembre del 2002, que en lo referente a industria señala como pequeñas y medianas empresas a las que tienen a partir de 11 trabajadores y hasta 250 ).

$\mathrm{Al}$ efectuar la prueba piloto, se apreció que determinadas preguntas del cuestionario no fueron bien recibidas por los empresarios, especialmente las referentes a la información financiera, datos del personal e inclusive datos generales, por lo que se suavizaron; de igual manera, se modificó la redacción en las preguntas concernientes a la integración vertical y horizontal para facilitar la comprensión.

Es pertinente comentar que no se obtuvo muestra porque el universo de las empresas pequeñas y medianas asciende a aproximadamente a un número de 60 , con una imprecisión causada por la inexactitud de los padrones disponibles; debido a ello, se decidió efectuar un censo del sector.

De igual manera, se tomó en cuenta que las empresas tienen una gran rotación de personal, por lo que frecuentemente pasan de ser microempresas (menos de 10 tra- 
bajadores) a ser pequeñas empresas (10 o más trabajadores), así como el hecho de que frecuentemente cambian su producto de muebles a otros productos derivados de la madera, como tarimas, cajas, empaques, etcétera. Una vez realizado lo anterior se aplicó el cuestionario ya modificado a 57 empresas, encontrando muy poca resistencia para proporcionar la información requerida.

Cuando se tuvieron contestados los cuestionarios se procedió a crear una base de datos en el programa SPSS (versión 12.0.). El propósito de lo anterior fue realizar las pruebas de confiabilidad, específicamente la prueba del Alfa de Cronbach. La fiabilidad, de acuerdo con Hair et al. (2004), es el grado de consistencia entre las múltiples medidas de una variable; por otro lado, la validación es la medida en la que una escala o conjunto de medidas representa con precisión el concepto de interés. En el cálculo del alfa de Cronbach si el valor que se obtiene después de aplicar la prueba es cercano a 1, existe un alto grado de explicación de las variables utilizadas en el cuestionario. En este caso, resultó que el valor del alfa es de 0.724 , es decir, ligeramente superior al mínimo requerido de 0.700; sin embargo, este valor puede incrementarse en la medida en que el instrumento se utilice en otras investigaciones subsecuentes y se vaya perfeccionando.

\section{Resultados}

En cuanto a la escala de medida de los datos, podemos afirmar en términos de Hair, et al. (2004), que se clasifica como observaciones métricas, de escala. De ahí que sobre la base de los mismos autores y de la relación de dependencia planteada en el objetivo del estudio, la técnica de análisis apropiada es la regresión múltiple. Cabe señalar que el uso de la técnica se orienta en esta investigación hacia fines de explicación, no de predicción.

Con respecto a la hipótesis particular 1: "Las pequeñas y medianas empresas del sector manufacturero forestal de la capital del estado de Durango utilizan la integración horizontal y/o vertical", se clasificó a las empresas por su tipo y grado de integración, mediante la variable "Tipo de integración", la cual se calculó para determinar el grado de empleo de cada una de las variables de la integración, por separado o conjuntamente, mediante las siguientes dimensiones: Ninguna integración, integración vertical, integración horizontal y ambas integraciones. 
Esta variable incluye a su vez las variables "Grado de integración vertical" y "Grado de integración horizontal", las cuales miden respectivamente el nivel de cada una de las variantes de la integración, basándose en los bloques del cuestionario que miden estas mismas. El análisis de frecuencias arroja los resultados descriptivos presentados a continuación:

Tabla 1

Tipos de integración

\begin{tabular}{|c|c|r|}
\hline Tipo de integración utilizada & Frecuencia & Porcentaje \\
\hline Ninguna integración & 38 & 66.67 \\
\hline Integración vertical & 14 & 24.56 \\
\hline Integración horizontal & 3 & 5.26 \\
\hline Ambas integraciones & 2 & 3.51 \\
\hline Total & 57 & 100.00 \\
\hline
\end{tabular}

Fuente: Elaboración propia

Sin embargo, es necesario saber si la diferenciación es estadísticamente significativa, para lo cual se recurrió a los datos de la base capturada en el programa SPSS versión 12.0 en español, con 57 observaciones en escala likert; un análisis de la varianza y una prueba F, misma que, de acuerdo con Lind, Marchal y Wathen (2005) se utiliza con el fin de verificar si las muestras provienen de poblaciones que tienen varianzas iguales con un nivel de confianza del 95\%; los resultados se muestran en la tabla 2:

Tabla 2

\section{Análisis de varianza con un factor}

\begin{tabular}{|c|c|c|c|c|c|c|}
\hline $\begin{array}{c}\text { Formas } \\
\text { de integración }\end{array}$ & $\begin{array}{c}\text { Fuente } \\
\text { de varianza }\end{array}$ & $\begin{array}{c}\text { Suma de } \\
\text { cuadrados }\end{array}$ & $\mathrm{gl}$ & $\begin{array}{c}\text { Media } \\
\text { cuadrática }\end{array}$ & $\mathrm{F}$ & Sig. \\
\hline \multirow{3}{*}{$\begin{array}{c}\text { Grado } \\
\text { de integración } \\
\text { horizontal }\end{array}$} & Inter-grupos & 13.587 & 3 & 4.529 & 13.853 & .000 \\
\hline & Intra-grupos & 17.328 & 53 & .327 & & \\
\hline & Total & 30.915 & 56 & & & \\
\hline \multirow{3}{*}{$\begin{array}{c}\text { Grado } \\
\text { de integración } \\
\text { vertical }\end{array}$} & Inter-grupos & 21.540 & 3 & 7.180 & 32.305 & .000 \\
\hline & Intra-grupos & 11.780 & 53 & .222 & & \\
\hline & Total & 33.319 & 56 & & & \\
\hline
\end{tabular}

Fuente: Elaboración propia 
En la primera columna se muestran las formas de integración; en la segunda, se ubican las fuentes de varianza; en la columna siguiente se muestra la suma de los cuadrados ya calculados; en la tercera, los grados de libertad asociados a la varianza; luego el cuadrado de las medias; después los resultados de la prueba F; y, por último, el grado de significación que define el peso de la evidencia contra la hipótesis nula.

Como se puede apreciar, la diferencia de media es significativa, lo cual indica que los grupos de integración horizontal y de integración vertical están plenamente identificados; no obstante, el de integración vertical está más diferenciado, esto es, sus características están más acentuadas.

Del análisis de medias, se concluye que las empresas que utilizan la integración vertical no son las mismas que utilizan la integración horizontal; esto es, que están diferenciadas, que hay evidencia estadística de que son grupos diferentes. Lo anterior permite suponer que las empresas que utilizan cualquiera de las modalidades de la integración no consideran, en la mayoría de los casos, utilizar la otra, y, también, que prefieren controlar el acceso a sus mercados y materias primas, es decir, su cadena de valor, antes que asociarse con sus competidores.

\subsection{Influencia de la integración en la rentabilidad}

Es pertinente aclarar que el análisis de regresión se presenta después de haberse efectuado un análisis de correlaciones canónicas, el cual arrojó resultados prácticamente iguales; entonces, siguiendo el principio de parsimonia, se presenta la alternativa más económica. De acuerdo con Hair et al. (1999:143), este análisis "es con mucho la técnica de dependencia más versátil y utilizada, aplicable en cualquier ámbito de los negocios". Además, es necesario mencionar que por razones de espacio se omiten las tablas con los valores de las variables.

En función de la hipótesis particular 2, se asume que existe una relación directa entre el grado de rentabilidad de una empresa en el mercado y el nivel de integración vertical y horizontal que presenta. Con base en lo anterior, el modelo por probar se define de la siguiente manera:

$$
\text { Yrent }=\alpha+\mathrm{b} 1 \mathrm{X}_{\mathrm{IV}}+\mathrm{b} 2 \mathrm{X}_{\mathrm{IH}}+\epsilon
$$


Donde:

Yrent $=$ Grado de rentabilidad de la empresa

$\alpha=$ Intercepto

$\mathrm{X}_{\mathrm{IV}}=$ Nivel de integración vertical

$X_{\mathrm{IH}}=$ Nivel de integración horizontal

$\epsilon=$ Término de error

b1, b2 $=$ Coeficientes asociados a las variables independientes

En primera instancia se procedió a validar los supuestos estadísticos inherentes al procedimiento de mínimos cuadrados sobre el que descansa el análisis de regresión; es decir normalidad, linealidad, homocedasticidad, multicolinealidad y autocorrelación.

Una vez efectuado el análisis se obtuvieron los siguientes resultados:

$$
\begin{aligned}
& \text { Yrent }=1.830+.213 \mathrm{X}_{\mathrm{IV}}-.115 \mathrm{X}_{\mathrm{IH}} \\
& (\mathrm{t}) \quad(2.327)(.948)(-.529) \\
& \mathrm{R}^{2}=.017
\end{aligned}
$$

Como se puede observar, y como resultado de todas las pruebas, fue estadísticamente insignificante el impacto de la integración sobre la rentabilidad de la empresa - Yrent - debido al impacto despreciable de los coeficientes y por los test estadísticos de dichos coeficientes.

De lo anterior, se concluye que no hay evidencia estadísticamente significativa que respalde una posible influencia de la integración en cualquier modalidad sobre la rentabilidad. Las empresas no reflejan que el uso o no de la integración beneficie o perjudique sus resultados.

\subsection{Influencia de la integración en la permanencia}

En función de la hipótesis particular 3, se asume que existe una relación directa entre el grado de permanencia de una empresa en el mercado, y el nivel de integración vertical y horizontal que presenta. Con base en lo anterior, el modelo por probar se define de la siguiente manera: 
Yperm $=\alpha+\mathrm{b} 1 \mathrm{X}_{\mathrm{IV}}+\mathrm{b} 2 \mathrm{X}_{\mathrm{IH}}+\epsilon$

Donde:

Yperm $=$ Grado de permanencia de la empresa

$\alpha=$ Intercepto

$\mathrm{X}_{\mathrm{IV}}=$ Nivel de integración vertical

$\mathrm{X}_{\mathrm{IH}}=$ Nivel de integración horizontal

$\in=$ Termino de error

b1, b2 = Coeficientes asociados a las variables independientes

En primera instancia se procedió a validar los supuestos estadísticos inherentes al procedimiento de mínimos cuadrados sobre el que descansa el análisis de regresión; es decir, normalidad, linealidad, homocedasticidad, multicolinealidad y autocorrelación.

La contrastación de supuestos, específicamente en cuanto a la homogeneidad de varianza y la normalidad de la distribución, sugirió la necesidad de transformar las variables dependiente e independiente. Después de varios intentos: inversa, raíz cuadrada y logaritmos, se confirmó que no había una mejora sustantiva respecto al ajuste del modelo original.

Dichos resultados se ven de la siguiente manera:

$$
\begin{aligned}
& \text { Yperm }=5.051-.054 \mathrm{X}_{\mathrm{IH}}-.217 \mathrm{X}_{\mathrm{IV}} \\
& (12.257)(-.454)(-1.911) \\
& \mathrm{R}^{2}=.097
\end{aligned}
$$

Como se puede observar, y como resultado de todas las pruebas, fue estadísticamente insignificante el impacto de la integración horizontal - XIH- sobre la permanencia de la empresa - Yperm. Lo anterior, se justifica tanto por el impacto despreciable de los coeficientes, por las pruebas estadísticos de dichos coeficientes, así como por el procedimiento de incluir variable a variable en el modelo en función del ajuste total. Incluso, con un nivel de significancia del 95\%, en el modelo anterior parece que el grado de integración vertical tampoco es significativa, aunque se encuentra prácticamente en el límite de la zona de rechazo $(\mathrm{t}=-1.911)$. 
Por lo anterior, el modelo final únicamente incluye a la integración vertical como variable explicativa del grado de permanencia. Los resultados finales fueron:

$$
\begin{aligned}
& \text { Yperm }=4.930-.24 \mathrm{X}_{\mathrm{IV}} \\
& (15.826)(-2.383) \\
& \mathrm{R}^{2}=.094
\end{aligned}
$$

Como puede observarse en el modelo final, resulta estadísticamente significativo el impacto de la variable independiente: nivel de integración vertical, sobre el grado de permanencia de la empresa. No obstante, es preciso reconocer que el ajuste del modelo es muy pobre; es decir, el comportamiento de los distintos niveles de permanencia de la empresa es apenas explicada en un $9.4 \%$ por la variable independiente. Podría pensarse que esta relación directa implica que las empresas que controlan su cadena de valor permanecen más en el tiempo que las que no; sin embargo, la fuerza de esta correlación es muy débil, casi nula.

\section{Conclusiones}

Las respuestas que se han obtenido indican, en el primer caso, que las Pymes muebleras de la ciudad de Durango utilizan alguna forma de integración en el 33.33\% de los casos.

La integración vertical es utilizada por el $24.56 \%$ de las mismas y, en cambio, la integración horizontal solamente es empleada por un 5.26\%. La combinación de ambos tipos de integración es utilizada por el $3.51 \%$ de las empresas.

En relación con la influencia de la integración en la rentabilidad (pregunta e hipótesis 2), se aprecia que no hay evidencia estadística de que ninguna de las dos modalidades de la integración tenga influencia en la rentabilidad de las empresas. En el caso de la influencia de la integración en la permanencia de las empresas (pregunta e hipótesis 3), sí se aprecia una débil influencia de la integración vertical en la permanencia de las empresas. Esto se entiende en el sentido de que las empresas que van realizando inversiones a lo largo del tiempo para expandirse a lo largo de su cadena de valor, van controlando su abasto de materia prima y sus mercados, lo cual incide positivamente en su estabilidad y, en último caso, en su permanencia cronológica, aunque se insiste en la debilidad de esta correlación. 
Si bien la Teoría de la Firma nos explica cómo en ciertos casos las empresas reemplazan los costos del mercado fabricando ellas mismas, en este caso se cumple lo establecido por Quinn, Dorley y Paquette (1990), quienes encuentran evidencia empírica de que en un medioambiente turbulento no siempre es bueno integrarse verticalmente, pues se dificulta la respuesta frente a cambios muy rápidos.

Se puede recomendar a los empresarios fabricantes de muebles incrementar el uso de la integración como área de oportunidad para definir estrategias que aumenten la ventaja competitiva, por las ventajas teóricas que esto implica, (ver Gil, 2005, Mpoyi, 2003, Galetovic, 2003, Economides, 1998), especialmente en lo concerniente al fomento del asociacionismo que fortalece la integración horizontal (ver Gómez López, 2000).

Es también posible recomendar a los académicos la medición de la integración en diversos escenarios y en diversas industrias, con el fin de verificar el poder descriptivo de la teoría y evaluar la conveniencia de integrar las modalidades vertical y horizontal.

\section{Bibliografía}

CaI, Hongbin y Ichiro Obara (2005), Firm Reputation And Horizontal Integration, Department of Economics, UCLA.

Colangelo, Giuseppe (1995), "Vertical vs. Horizontal integration: pre-emptive merging", The Journal of Industrial Economics, Vol. XLIII, No. 3, septiembre, pp. 323-337.

CoMPÉs LóPEZ, Raúl (2005), "La desintegración vertical del transporte terrestre en España”, Revista de Economia Institucional, Vol. 7, No. 13.

Dallago, Bruno (2003), "The importance of Small and Medium Enterprises", UN Chronicle, United Nations Publications, No. 4, EEUU, p. 18.

Diario Oficial de la Federación, 30 de diciembre de 2002.

ECONOMides, Nicholas (1998), Competition and Vertical Integration in the Computing Industry, Stern School of Business, NY, EEUU. 
Galetovic, Alexander (2003), Integración vertical en el sector eléctrico: una guía para el usuario, Centro de Economía Aplicada, Universidad de Chile.

GIL, Ricard (2005), Decision Rights and Vertical Integration in the Movie Industry, University of California, Santa Cruz.

GómEz LóPEZ, Claudia Susana (2000), “Alianzas estratégicas tecnológicas en la industria química mexicana”, Revista Mexicana de Sociología, Año LXII, No. 3, julio, pp. 119-144.

Hair, Joseph F. et al. (2004), Análisis multivariante, Pearson Prentice Hall, España.

Hernández Romo, Marcela (2004), La cultura empresarial en México, Universidad Autónoma de Aguascalientes, México.

Hill, Charles W. y Gareth R. Jones (2005), Administración estratégica: un enfoque integrado, McGraw Hill, México.

Lind, Douglas A., William G. Marchal y Samuel A. Wathen (2005), Estadística aplicada a los negocios y a la economía, 12a. ed., Mc Graw Hill, México.

MintzBerg, Henry y James Brian Quinn (1993), El proceso estratégico, Prentice Hall, México.

Mpoyi, Richard T. (2003), "Vertical Integration: Strategic Characteristic And Competitive Implications", Competitiveness Review, Vol. 13, No. 1, pp. 44-55.

Paredes Molina, Ricardo (1997), "Integración vertical: Teoría e implicancias de política pública”, Estudios Públicos, No. 66.

Porter, Michael E. (1990), "The Competitive Advantage of Nations: Cover Story", Harvard Business Review, No. 2, pp 73-93.

Puig-Junoy, Jaume y P. Perez-Sust (2001), "Integración vertical y contratación externa en los servicios generales de los hospitales españoles”, Gaceta Sanitaria, Vol. 1, No. 2.

Quinn, J. B., T. L. Doorley y P.C. Paquette (1990), “Technology in Services: Rethinking Strategic Focus", MIT Sloan Management Review, Vol. 31, No. 2, pp. 79-87.

Rojas Rodríguez, Freddy (1995), "Integración bosque industria: Una necesidad regional", Madera y Bosques, Vol. 5, No. 5. 
SCAZZIERI, Roberto (1990), "Vertical Integration in economic theory", Journal of Postkeynesial Economics, Otoño, Vol. 13, No. 1.

SeCRetaría DE ECONOMíA (junio, 2004), http://www.siemb.gob.mx/portalsiem/estadisticas/repsector (A) 